\title{
Estimation of Heterosis and Combining Ability Effects on Grain Yield and Some Agronomic Traits of Sorghum under Three NPK Fertilizers Levels
}

\author{
M.A. Sayed ${ }^{\#}$ and M.T. Said \\ Agronomy Department, Faculty of Agriculture, Assiut University, \\ Assiut, Egypt.
}

\begin{abstract}
ETEROSIS and combining ability for grain yield and some agronomic traits were studied among thirty $\mathrm{F} 1$ grain sorghum crosses and their elven parents under three NPK levels during 2014 and 2015 seasons. Significant differences among genotypes were found for all studied traits, indicating wide genetic diversity. The interaction of genotypes with each of years and NPK levels were significant in most studied traits. The analysis of variance for combining ability revealed that the mean square due to entries, parents, parents vs. crosses, crosses, lines, testers, lines $\times$ testers turned up significant for all studied characters and suggesting that the experimental materials possessed considerable variability that both general and specific combining ability were involved in the genetic expression of these characters. The female line ICSB610 showed significant and negative general combining ability (GCA) effects for days to $50 \%$ heading and panicle length and positive for grain yield and plant height. It may be used to develop high yielding, early flowering, and tall hybrids with short panicles. For specific combining ability (SCA), effects, the crosses ICSA613 × ICSR89028 and ICSA20 $\times$ ICSR53 gave positive and highly significant SCA effects which indicated that these crosses can be considered desirable combiners. These crosses had also high grain yield per se and one of the parents with highest GCA effects. The observations on portioning of combining ability variance into additive and dominance variances indicated the role of both additive and dominance gene action. The magnitude of non-additive variance was higher than the additive variance by many folds for all studied traits.
\end{abstract}

Keywords: Heterosis, Combining ability, Sorghum, NPK nutrients

Grain sorghum (Sorghum bicolor L. Moench) is the fifth leading cereal crop in the world after maize, rice, wheat, and barley (FAOSTAT, 2014). However, sorghum grain is the staple food of poor and the most food-insecure people, living mainly in the semi-arid regions (Ali et al., 2009 and Bibi et al., 2010). Africa contributes more than $60 \%$ of the total land area under sorghum (FAOSTAT, 2012), but the yields have remained low (less than $1 \mathrm{t} \mathrm{ha}^{-1}$ ) due to continuous use of low yielding cultivars (Ringo et al., 2015). Sorghum performs better under adverse soil and weather conditions as compared to other crops (Ejeta \& Knoll, 2007). Worldwide, crop production is restricted by the 
concentrations and chemical forms of mineral elements, and adequate supplies of the essential mineral elements nitrogen $(\mathrm{N})$, potassium $(\mathrm{K})$, phosphorus $(\mathrm{P})$ and the other essential mineral elements are required for maximal crop production (White \& Brown, 2010). Phytoavailability of N, K, P, or S often limits low-input agriculture (Fageria et al., 2011 and Mueller et al., 2012). Moreover, sorghum is a C4 annual crop which can produce high forage biomass yields per unit of area (Rooney et al., 2007) and uses nitrogen in a more efficient way compared to most C3 crops (Young \& Long, 2000). Sorghum was investigated under low fertility conditions by several studies (Al-Naggar et al., 2006; Hovny \& ElDsouky, 2007; Abd EL-Mottaleb, 2009; Omar et al., 2014 and Amir \& Mohamed, 2015). They stated that reducing $\mathrm{N}$ levels delayed flowering date, reduced grain yield plant $^{-1}$ and reduced 1000 grain weight as well as significant positive GCA effects of female and male lines under low nitrogen level for grain yield plant ${ }^{-1}$ and 1000 grain weight. Utilization of grain sorghum hybrids can significantly increase yields in sorghum growing areas (House et al., 1997) because they out-yield local cultivars and improved varieties by $20-60 \%$ (Bantilan et al., 2004). This potential of hybrids is estimated from the percentage increase or decrease of their performance over the mid-parent (average heterosis) and better-parent (heterobeltiosis) (Hochholdinger \& Hoecker, 2007). Furthermore, both types of heterosis were worked out in order to have broad picture of performance for materials across dry lands and sub-humid environments, and positive heterosis in a desired trend is preferred in selection for yield and its components (Lamkey \& Edwards, 1999). Combining ability analysis is one of the powerful tools available to estimate the combining ability effects and aids in selecting the desirable parents and crosses for the exploitation of heterosis (Sarker et al., 2002 and Rashid et al., 2007). The general combining ability (GCA) of parental genotypes should be examined when the objective is the development of superior genotypes, while the specific combining ability (SCA) effects provide information about the performance of hybrids (Cruz \& Regazzi, 1994). The differences in GCA are mainly due to the additive genetic effects and higher order additive interactions, while the differences in SCA are attributed to the non-additive dominance and other types of epitasis (Falconer, 1989). This information would be useful to investigate the performance and relationship of F1 hybrids and parents and to select suitable parents and population for designing an effective breeding program. Presence of heterosis, GCA and SCA effects for yield and its related traits are reported by Abo-Elwafa et al. (2005), Faiz et al. (2006), Hovny \& El-Dsouky (2007), Saleem et al. (2008), Abd eL-Mottaleb (2009), Kanbar et al. (2011) and Omar et al. (2014).

This investigation aimed to: 1) Determine heterosis over mid- and betterparent for yield and agronomic traits by identifying suitable heterotic parents under NPK levels. 2) Assess the combining ability of current sorghum materials, aiming to a parental selection improvement for low NPK fertility tolerance in breeding programs to increase production. 


\section{Materials and Methods}

Plant materials and hybrids development

Plant material of the experiment comprised of $30 \mathrm{~F}_{1}$ grain sorghum crosses formed by crossing six inbred lines (cytoplasmic male sterility lines) to five testers in a line $x$ tester mating design in the summer season of 2013 to generate the breeding material and two standard checks (Hybrid 305 and Dorado). The female lines (ICSA20, ICSA52, ICSA608, ICSA610, ICSA613 and ICSA93) and male lines (ICSR29, ICSR53, ICSR89028, ICSR91020 and ICSR93004) were obtained from India (International Crop Research Institute for Semi-Arid Tropics, ICRISAT).

\section{Experimental site}

The 43 grain sorghum entries were grown at Assiut Agricultural Research Farm, Assiut University, Assiut, Egypt. The preceding crop of the experimental site for the two seasons was wheat. The physical and chemical properties of experimental site are shown in Table 1.

TABLE 1. Some physical and chemical properties of representative soil samples of the experimental site before sowing $(0-30 \mathrm{~cm}$ depth) for the two growth seasons.

\begin{tabular}{|c|c|c|}
\hline Soil property & 2010/2011 season* & 2011/2012 season* \\
\hline \multicolumn{3}{|l|}{ Particle - size distribution } \\
\hline Silt (\%) & 27.4 & 27.3 \\
\hline Sand $(\%)$ & 24.3 & 25.2 \\
\hline Clay $(\%)$ & 48.3 & 47.5 \\
\hline Texture & Clay & Clay \\
\hline Organic matter $(\%)$ & 1.75 & 1.72 \\
\hline Field capacity $(\%)$ & 42.8 & 43.2 \\
\hline EC $(1: 1$ extract $)\left(\mathrm{dS} \mathrm{m}^{-1}\right)$ & 0.74 & 0.77 \\
\hline $\mathrm{pH}(1: 1$ suspension $)$ & 8.2 & 8.1 \\
\hline Total nitrogen $(\%)$ & 0.72 & 0.69 \\
\hline $\mathrm{CaCO}_{3}(\%)$ & 3.4 & 3.5 \\
\hline KCl-extractable $\mathrm{N}\left(\mathrm{mg} \mathrm{kg}^{-1}\right)$ & 41.23 & 40.26 \\
\hline $\mathrm{NaHCO}_{3}$-extractable $\mathrm{P}\left(\mathrm{mg} \mathrm{kg}^{-1}\right)$ & 4.36 & 4.65 \\
\hline $\mathrm{NH}_{4} \mathrm{OAC}$-extractable $\mathrm{K}\left(\mathrm{mg} \mathrm{kg}^{-1}\right)$ & 49.24 & 50.86 \\
\hline
\end{tabular}

* Each value represents the mean of three replications.

\section{Experimental design and field management}

The field design was a randomize compete block design (RCBD) using strip plot arrangement with three replicates. NPK levels were allocated to the main plots and entries to subplots. Each entry (genotype) was placed in a three rows plot of $3 \mathrm{~m}$ long and $60 \mathrm{~cm}$ apart with $20 \mathrm{~cm}$ between plants. Trial was hand 
planted with 3-4 seeds per hill, which was later be thinned to secure two plants per hill. Planting was done in the two summer successive seasons at $17^{\text {th }}$ and $16^{\text {th }}$ of June in 2014 and 2015 seasons, respectively. Standard cultural practices for optimum sorghum production were carried out in both seasons. Three fertilizers levels, $100 \%$ (L1), $75 \%$ (L2) and $50 \%$ (L3) of the recommended doses, i.e. 240, 54 and $57.6 \mathrm{~kg} / \mathrm{ha}$ of $\mathrm{N}, \mathrm{P}$ and $\mathrm{K}$ fertilizers, respectively. Urea $(46.5 \% \mathrm{~N})$, Superphosphate $\left(15 \% \mathrm{P}_{2} \mathrm{O}_{5}\right)$ and Potassium sulphate $\left(48 \% \mathrm{~K}_{2} \mathrm{O}\right)$ were used as a source of $\mathrm{N}, \mathrm{P}$ and $\mathrm{K}$, respectively. The super-phosphate and potassium sulfate were applied once before the first irrigation, while urea was divided into three doses and applied before the first, second and third irrigations.

\section{Data collection}

The data was recorded for days to $50 \%$ heading (HD; day) on whole plot basis, whereas plant height $(\mathrm{PH} ; \mathrm{cm})$ and panicle length $(\mathrm{PL} ; \mathrm{cm})$ on the average samples of five random competitive plants from each genotype were tagged in each replication (border plants were excluded). Seed index (SI; g) was recorded on the weight of 1000-grain in grams from each genotype per each replication, while, biological yield (BY; $\mathrm{t} \mathrm{ha}^{-1}$ ) and grain yield ( $\mathrm{GY} ; \mathrm{t} \mathrm{ha}^{-1}$ ) were recorded on the total number of plants per plot, then the data of biological yield and grain yield per plot were transformed to $\mathrm{t} / \mathrm{ha}$.

\section{Statistical analysis and procedures}

Analysis of Variance

$30 \mathrm{~F}_{1}$ hybrids, their parents and two check varieties were evaluated for grain yield and some agronomic traits over three NPK levels and two years. Years (Y) and NPK levels represent six different environmental conditions, according to this, the combined analysis was performed according to Steel et al. (1997) after carrying out the homogeneity using Bartlett test, to estimate the main effects of the different sources of variation and their interactions.

\section{Correlation}

Phenotypic correlations among studied traits were determined under overall NPK levels and years using Pearson's correlation test. The ANOVA and the correlation test were performed using SAS software (v 9.2, 2008).

Combining ability analysis

The general (GCA) and specific (SCA) combining ability of the parents and hybrids were worked out as per the method outlined by Kempthorne (1957) in order to determine the significance of differences among hybrids and parents.

$$
\begin{aligned}
& \text { Heritability } \\
& \text { Broad }\left(H^{2}{ }_{B}\right) \text { and narrow }\left(H^{2}{ }_{N}\right) \text { sense heritability were measured as follows: } \\
& \qquad H^{2}{ }_{B}=\sigma_{G}^{2} / \sigma_{P}^{2}
\end{aligned}
$$

Egypt. J. Agron. 38, No. 2 (2016) 
where, $\sigma_{G}^{2}$ is the genetic variance, $\sigma_{P}^{2}$ is the phenotypic variance and $\sigma_{A}^{2}$ is the additive variance.

\section{Heterosis}

Mid-parent (MP) and best-parent (BP) heterosis percentages were computed by using the following fomulas:

$$
M P_{i j}=\frac{F_{1 i j}-M p_{(F 1 i j)}}{M p_{(F 1 i j)}} \quad B P_{i j}=\frac{F_{1 i j}-B P_{(F 1 i j)}}{B P_{(F 1 i j)}}
$$

where $\mathrm{MP}_{\mathrm{ij}}$ is the heterosis of the $i j$ th cross; $\mathrm{BP}_{\mathrm{ij}}$ is the heterobeltiosis (bestparent heterosis) of the ijth cross; $\mathrm{F}_{1 \mathrm{ij}}$ is the mean of the $i j$ th $\mathrm{F}_{1}$ cross; $\mathrm{MP}\left(\mathrm{F}_{1 \mathrm{ij}}\right)$ is the mid-parent [(Parent1 + Parent2) / 2] for the ${ }_{\mathrm{ij}}$ th cross; and $\mathrm{BP}\left(\mathrm{F}_{1 \mathrm{ij}}\right)$ is the best parent values for the ijth cross. Significance was tested by the appropriate revised Least Significant Difference (LSD) at 5\% level of significant according to Steel \& Torrie (1981).

\section{Results and Discussion}

Data in Table 2 show that all the variance components, years, NPK levels, genotypes and interactions were affected significantly in all studied traits except plant height $(\mathrm{PH})$ and biological yield which were not affected significantly by the NPK levels and years, respectively. Moreover, significant differences among genotypes were found for all studied traits, indicating wide genetic diversity. The interaction of genotypes with each of years and NPK levels were significant in most studied traits, reflecting that expression of these traits are controlled mostly by the non-additive effects of genes that are not stable under NPK levels over both years. Similarly, the analysis of variance for combining ability revealed that the mean square due to entries, parents, parents vs. crosses, crosses, lines, testers, lines $\times$ testers turned up significant for all studied characters suggesting that the experimental materials possessed considerable variability in both general and specific combining ability involved in the genetic expression of these characters (Table 3). The results obtained were concur with those obtained by Al-Nagar et al. (2007) and Abou-Amer \& Kewan (2014) who found that N and NP levels and their interaction with genotypes had a highly significant effect on sorghum green fodder yield and grain yield $\left(\mathrm{t} / \mathrm{fad}\left(\mathrm{fad}=2400 \mathrm{~m}^{2}\right)\right)$. Variation among parents, crosses, parents vs crosses, lines, testers and line $\times$ testers also was observed in several sorghum studies such as Hovny \& El-Dsouky (2007), Abdel-Mottaleb (2009), Essa (2009) and Mahdy et al. (2011). 
TABLE 2. Mean squares of the combined analysis of variance for grain yield and agronomic traits.

\begin{tabular}{|l|c|c|c|c|c|c|c|}
\hline \multicolumn{1}{|c|}{ S.V. } & DF & $\mathbf{5 0 \%}$ HD & PH; cm & PL; cm & SI; g & BY; T/H & GY; T/H \\
\hline Year (Y) & 1 & 2449.8 & 35710.02 & 140.70 & 1308.14 & 5034.68 & 5.78 \\
Y(Rep) & 4 & 9.30 & 477.92 & 35.68 & 28.08 & 959.33 & 0.57 \\
\hline NPK levels (L) & 2 & 274.21 & 1674.27 & 217.83 & 118.89 & 1244.41 & 134.12 \\
YL & 2 & 96.42 & 324.61 & 197.65 & 42.04 & 31.72 & 1.29 \\
YL(Rep) & 8 & 7.21 & 456.25 & 8.53 & 11.68 & 31.50 & 0.34 \\
\hline Genotypes (G) & 42 & 195.59 & 7773.13 & 90.35 & 68.74 & 3284.03 & 17.57 \\
GY & 42 & 73.32 & 2035.31 & 36.95 & 35.50 & 220.58 & 16.70 \\
GL & 84 & 4.68 & 77.99 & 7.76 & 10.38 & 82.84 & 1.23 \\
GYL & 84 & 5.42 & 104.00 & 7.98 & 10.79 & 14.47 & 1.09 \\
Error & 504 & 1.72 & 104.55 & 5.99 & 7.95 & 71.94 & 0.38 \\
\hline
\end{tabular}

; ${ }^{* * *}$ Significant at the 0.05 and 0.01 probability levels, respectively

TABLE 3. Mean squares of the combined line $\times$ tester analysis for all studied traits overall environments (NPK levels and the two years).

\begin{tabular}{|c|c|c|c|c|c|c|c|}
\hline Source & DF & $\begin{array}{l}50 \% \\
\text { HD }\end{array}$ & PH; cm & PL; cm & SI; $\mathbf{g}$ & BY; T/H & GY; T/H \\
\hline $\begin{array}{l}\text { Environments } \\
\text { (Envi) }\end{array}$ & 5 & $626.8^{* *}$ & $7766.8^{* * *}$ & $187.5^{* *}$ & $318.2^{* *}$ & $1455.9^{*}$ & $54.5^{* *}$ \\
\hline Rep(Envi) & 12 & $8.2^{* *}$ & $423.2^{* *}$ & $17.1^{* *}$ & $14.7^{*}$ & $392.2^{* *}$ & 0.5 \\
\hline Entries $(E)$ & 40 & $191.9^{* *}$ & $6951.8^{* *}$ & $89.4^{* *}$ & $69.5^{* * *}$ & $3301.3^{* *}$ & $17.7^{* * *}$ \\
\hline Parents $(\mathrm{P})$ & 10 & $348.2^{* *}$ & $2217.6^{* *}$ & $92.6^{* *}$ & $129.2^{* * *}$ & $2903.3^{* *}$ & $5.3^{* *}$ \\
\hline $\mathrm{P}$ vs $\mathrm{C}$ & 1 & $41.8^{* *}$ & $200523^{* * *}$ & $1383.2^{* * *}$ & $239.6^{* *}$ & $76785.4^{* *}$ & $482.4^{* *}$ \\
\hline Crosses (C) & 29 & $143.2^{* *}$ & $1909.4^{* *}$ & $43.7^{* *}$ & $43.0^{* *}$ & $904.6^{* *}$ & $5.9^{* *}$ \\
\hline Lines (L) & 5 & $80.8^{* *}$ & $1885.3^{* *}$ & $43.8^{* *}$ & $112.4^{* * *}$ & $1587.3^{* *}$ & $25.7^{* * *}$ \\
\hline Testers $(\mathrm{T})$ & 4 & $573.5^{* *}$ & $5435.8^{* *}$ & $94.5^{* *}$ & $90.6^{* *}$ & $3414.3^{* *}$ & $39.8^{* * *}$ \\
\hline $\mathrm{L}^{*} \mathrm{~T}$ & 20 & $72.7^{* *}$ & $1210.2^{* *}$ & $33.5^{* *}$ & $16.1^{* * *}$ & $231.9^{* *}$ & $15.2^{* * *}$ \\
\hline Envi $^{*} \mathrm{E}$ & 200 & $19.1^{* *}$ & $499.7^{* *}$ & $13.8^{* *}$ & $15.3^{* *}$ & 86.5 & $4.4^{* * *}$ \\
\hline Envi* $^{*} \mathrm{P}$ & 50 & $15.7^{* *}$ & $605.5^{* *}$ & $13.4^{* *}$ & $21.1^{* * *}$ & 35.9 & $5.6^{* *}$ \\
\hline P vs C "Envi & 5 & $7.1^{* *}$ & 213.5 & $24.2^{* * *}$ & 8.5 & $92.3^{*}$ & $86.7^{* *}$ \\
\hline Envi $^{*} \mathrm{C}$ & 145 & $20.7^{* *}$ & $473.0^{* *}$ & $13.7^{* *}$ & $13.5^{* *}$ & $103.7^{* *}$ & $1.2^{* *}$ \\
\hline Envi* ${ }^{*}$ & 25 & 15.4 & 584.2 & $19.9^{* *}$ & $20.6^{*}$ & 77.0 & $9.7^{* *}$ \\
\hline Envi $^{*} \mathrm{~T}$ & 20 & $49.0^{* * *}$ & 590.4 & $21.2^{* *}$ & 16.2 & $393.5^{* *}$ & $3.8^{* * *}$ \\
\hline Envi ${ }^{*} \mathrm{~L}^{*} \mathrm{~T}$ & 100 & $16.4^{* *}$ & $421.8^{* *}$ & $10.6^{* *}$ & $11.3^{* *}$ & 52.4 & $2.7^{* *}$ \\
\hline Error & 480 & 1.8 & 107.5 & 6.1 & 7.5 & 71.8 & 0.37 \\
\hline
\end{tabular}

;" 
Days to $50 \%$ heading

As an average of the genotypes (Table 4), heading date was delayed by decline the amount of NPK fertilizers added. Since, significant difference between L1 (69.7 day) and L3 (71.7 day) was observed. Similar result was observed by Omar et al. (2014) who found that mean days to $50 \%$ flowering of hybrids and their parents were increased by increasing nitrogen stress. Under the three NPK levels, female lines and crosses were earlier than male lines and check varieties. About half of the crosses were earlier than the earliest check variety (Hybrid 305), and the last was earlier than Dorado cultivar. The results furthermore, reveal that elven crosses exhibited significant and negative midparent heterosis (Table 5). Also, elven crosses show highly significant and negative specific combining ability (SCA) indicating that these crosses can be considered as good combiners for earliness. Among the parental lines, five lines show negative and highly significant general combining ability (Table 6). The cross (ICSA610 $\times$ ICSR29) shows significant and negative mid-parent heterosis and had highest negative SCA values among crosses (Table 7). The female line ICSB610 had the highest negative GCA among females and involved in the best cross in days to $50 \%$ heading. The previous results indicated that this line may be harboring the genes that underlay the earliness, whereas in testers, ICSR91020 gave maximum GCA effect $\left(-3.76^{* *}\right)$, these parents contributed to improving short duration to heading in the crosses. These results are in harmony with those obtained by Hovny \& El-Dsouky (2007), Abdel-Mottaleb (2009), Essa (2009), Mahdy et al. (2011), Abou-Amer \& Kewan (2014), Omar et al. (2014) and Amir \& Mohamed (2015).

\section{Plant height (PH)}

The plant height of sorghum genotypes decreased as a result of reducing the amount of NPK fertilizers comparing with $100 \%$ of recommended NPK. The average reduction in plant height was not significant. However, the majority of the crosses were taller than their parents and the check verities indicating the existence of heterosis under all studied NPK levels. As an average, the maximum plant height $(204.5 \mathrm{~cm})$ was set by the cross ICSA608 $\times$ ICSR29 followed by ICSA613 $\times$ ICSR53 $(200.8 \mathrm{~cm})$ as per se hybrid performance (Table 4). Among female lines, ICSB93 set the tallest plant height $(156.0 \mathrm{~cm})$ whereas in testers, ICSR53 gave the highest value of plant height $(162.9 \mathrm{~cm})$. Line ICSB613 gave maximum $\left(6.03^{* *}\right)$ positive GCA effects and involved in the second taller cross (Table 6), this line tends to increase plant height. Whereas among the testers, ICSR29 manifested maximum (10.00**) positive GCA effects and involved in the second taller cross indicating that both the parents retain more additive genes, thus may be utilized in hybridization programs for improving plant height in segregating population. The crosses (ICSA608 $\times$ ICSR29) and (ICSA52 $\times$ ICSR89028) gave maximum SCA effects of 12.06 and 11.74, respectively, which may be considered suitable for hybrid crop development (Table 7). These findings are in agreement with those obtained by Hovny \& El-Dsouky (2007), Abdel-Mottaleb (2009), Essa (2009), Mahdy et al. (2011) and Amir \& Mohamed (2015). 
TABLE 4. Mean performance of the $30 \mathrm{~F} 1$ hybrids. their respective parents and check varieties under three NPK levels (100, 75 and $50 \%$ ) over both years for days to $50 \%$ heading, plant height and panicle length.

\begin{tabular}{|c|c|c|c|c|c|c|c|c|c|c|c|c|}
\hline \multirow{2}{*}{\begin{tabular}{|c|} 
Traits \\
Genotypes \\
Irrigation \\
Hybrids \\
\end{tabular}} & \multicolumn{4}{|c|}{$50 \% \mathrm{HD}$} & \multicolumn{4}{|c|}{ PH; cm } & \multicolumn{4}{|c|}{ PL; cm } \\
\hline & $100 \%$ & $75 \%$ & $\mathbf{5 0 \%}$ & Mean & $100 \%$ & $75 \%$ & $\mathbf{5 0 \%}$ & Mean & $100 \%$ & $75 \%$ & $\mathbf{5 0 \%}$ & Mean \\
\hline ICSA20 $\times$ ICSR29 & 70.5 & 70.8 & 72.0 & 71.1 & 198.1 & 203.6 & 196.1 & 199.3 & 30.4 & 28.9 & 29.4 & 29.6 \\
\hline ICSA20 $\times$ ICSR53 & 71.3 & 72.2 & 74.7 & 72.7 & 173.8 & 178.3 & 174.2 & 175.4 & 30.4 & 27.5 & 28.6 & 28.8 \\
\hline ICSA20 × ICSR89028 & 71.0 & 71.8 & 73.2 & 72.0 & 173.3 & 165.1 & 165.3 & 167.9 & 31.9 & 30.6 & 30.6 & 31.0 \\
\hline ICSA20 $\times$ ICSR91020 & 66.8 & 67.7 & 69.3 & 67.9 & 175.9 & 177.1 & 177.2 & 176.7 & 32.2 & 30.7 & 29.9 & 30.9 \\
\hline ICSA20 × ICSR93004 & 74.2 & 74.5 & 75.2 & 74.6 & 184.7 & 183.3 & 169.7 & 179.2 & 33.1 & 29.2 & 26.8 & 29.7 \\
\hline ICSA52 $\times$ ICSR 29 & 73.3 & 72.2 & 75.3 & 73.6 & 183.2 & 185.8 & 181.4 & \begin{tabular}{|l|l|}
183.5 \\
\end{tabular} & 31.8 & 29.8 & 27.7 & 29.8 \\
\hline ICSA52 $\times$ ICSR53 & 68.7 & \begin{tabular}{|l|l}
69.8 \\
\end{tabular} & 71.7 & 70.1 & 186.4 & 185.2 & 183.1 & 184.9 & 27.5 & 25.1 & 26.0 & 26.2 \\
\hline ICSA52 $\times$ ICSR89028 & 69.2 & 68.5 & 70.0 & 69.2 & 198.6 & 191.7 & 189.3 & 193.2 & 31.9 & 29.7 & 30.9 & 30.8 \\
\hline ICSA52 $\times$ ICSR91020 & 65.3 & 66.5 & 67.3 & 66.4 & 180.8 & 179.8 & 181.6 & 180.7 & 28.7 & 26.5 & 25.6 & 26.9 \\
\hline ICSA52 × ICSR93004 & 67.5 & 69.3 & 70.3 & 69.0 & 171.9 & 173.8 & 165.9 & 170.5 & 30.8 & 30.9 & 30.7 & 30.8 \\
\hline ICSA608 × ICSR29 & 70.2 & 69.7 & 72.3 & 70.7 & 210.9 & 206.6 & 196.1 & 204.5 & 30.3 & 29.5 & 31.7 & 30.5 \\
\hline ICSA608 × ICSR53 & 69.3 & 70.2 & 72.3 & 70.6 & 184.2 & 183.0 & 179.4 & 182.2 & 31.8 & 26.9 & 29.3 & 29.3 \\
\hline ICSA608 × ICSR89028 & 71.5 & 71.3 & 74.7 & 72.5 & 182.5 & 181.0 & 175.6 & 179.7 & 32.6 & 31.4 & 31.0 & 31.7 \\
\hline ICSA608 × ICSR91020 & 64.0 & 65.8 & 67.0 & 65.6 & 177.1 & 172.6 & 166.1 & 171.9 & 32.3 & 29.9 & 31.3 & 31.2 \\
\hline ICSA608 × ICSR93004 & 75.5 & 76.8 & 75.3 & 75.9 & 182.0 & 173.4 & 166.8 & 174.1 & 30.2 & 28.4 & 29.4 & 29.3 \\
\hline ICSA610 $\times$ ICSR 29 & 65.3 & 66.3 & 68.2 & 66.6 & 195.8 & 190.2 & 192.6 & 192.9 & 30.6 & 27.8 & 30.2 & 29.5 \\
\hline ICSA610 $\times$ ICSR53 & 68.5 & 70.2 & 72.3 & 70.3 & 187.5 & 186.4 & 180.9 & 184.9 & 27.8 & 26.8 & 28.3 & 27.6 \\
\hline ICSA610 $\times$ ICSR 89028 & 70.7 & 72.2 & 74.7 & 72.5 & 180.7 & 176.3 & 172.0 & 176.3 & 28.7 & 27.8 & 28.7 & 28.4 \\
\hline ICSA610 $\times$ ICSR91020 & 66.8 & 67.2 & 67.8 & 67.3 & 181.9 & 182.3 & 181.2 & 181.8 & 30.9 & 30.4 & 29.8 & 30.4 \\
\hline ICSA610 × ICSR93004 & 68.7 & 69.0 & 70.3 & 69.3 & 189.6 & 187.1 & 171.9 & 182.9 & 28.8 & 27.5 & 25.5 & 27.3 \\
\hline ICSA613 × ICSR29 & 71.3 & 70.2 & 72.5 & 71.3 & 190.4 & 189.6 & 183.6 & 187.9 & 29.3 & 27.3 & 27.1 & 27.9 \\
\hline ICSA613 $\times$ ICSR53 & 66.5 & 67.3 & 67.2 & 67.0 & 202.2 & 202.9 & 197.4 & 200.8 & 32.7 & 30.7 & 28.2 & 30.5 \\
\hline ICSA613 × ICSR89028 & 69.3 & \begin{tabular}{|c|}
69.8 \\
\end{tabular} & 72.8 & 70.6 & 194.9 & 189.0 & 183.1 & 189.0 & 32.3 & 29.3 & 29.3 & 30.3 \\
\hline ICSA613 × ICSR91020 & 64.8 & 66.7 & 68.8 & 66.8 & 191.9 & 193.6 & 191.4 & 192.3 & 32.9 & 30.6 & 29.4 & 31.0 \\
\hline ICSA613 × ICSR93004 & 71.0 & 74.3 & 73.0 & 72.8 & 159.8 & 178.2 & 168.1 & 168.7 & 27.9 & 30.2 & 30.2 & 29.4 \\
\hline ICSA93 $\times$ ICSR29 & 72.5 & 72.3 & 75.5 & 73.4 & 185.1 & 184.7 & 177.1 & 182.3 & 32.5 & 31.6 & 31.9 & 32.0 \\
\hline ICSA93 $\times$ ICSR53 & \begin{tabular}{|l|}
67.8 \\
\end{tabular} & 68.7 & 69.2 & 68.6 & 182.6 & 184.2 & 181.1 & 182.6 & 28.7 & 26.1 & 26.3 & 27.0 \\
\hline ICSA93 × ICSR89028 & 70.8 & 71.2 & 74.2 & 72.1 & 182.6 & 177.0 & 172.7 & 177.4 & 32.8 & 28.8 & 32.4 & 31.3 \\
\hline ICSA93 × ICSR91020 & 64.5 & 65.5 & 66.0 & 65.3 & 167.4 & 162.4 & 165.1 & 165.0 & 32.2 & 31.9 & 29.6 & 31.2 \\
\hline ICSA93 $\times$ ICSR93004 & 72.3 & 73.8 & 74.2 & 73.4 & 166.3 & 164.5 & 157.8 & 162.9 & 29.7 & 27.3 & 28.6 & 28.5 \\
\hline Hybrid's mean & 69.3 & 70.1 & 71.6 & 70.3 & 184.1 & 183.0 & 178.1 & 181.7 & 30.8 & 29.0 & 29.1 & 29.6 \\
\hline \multicolumn{13}{|l|}{ Female lines } \\
\hline ICSB20 & 68.0 & 68.3 & 67.2 & 67.8 & 132.5 & 135.0 & 132.4 & 133.3 & 27.1 & 25.9 & 27.1 & 26.7 \\
\hline ICSB52 & 65.5 & 65.0 & 67.8 & 66.1 & 148.1 & 143.0 & 145.1 & 145.4 & 25.1 & 26.0 & 25.8 & 25.6 \\
\hline ICSB608 & 67.3 & 67.7 & 68.2 & 67.7 & 133.0 & 133.9 & 129.6 & 132.2 & 26.7 & 22.5 & 27.5 & 25.6 \\
\hline ICSB610 & 67.5 & 68.0 & 69.3 & 68.3 & 129.8 & 129.8 & 132.7 & 130.8 & 26.9 & 27.4 & 26.3 & 26.9 \\
\hline ICSB613 & 68.5 & 67.7 & 70.5 & 68.9 & 133.8 & 132.1 & 131.8 & 132.6 & 25.1 & 23.6 & 23.2 & 24.0 \\
\hline ICSB93 & 65.3 & 66.2 & 68.8 & 66.8 & 153.9 & 152.2 & 161.9 & 156.0 & 27.2 & 24.9 & 27.1 & 26.4 \\
\hline Female's Mean & 67.0 & 67.2 & 68.6 & 67.6 & 138.5 & 137.7 & 138.9 & 138.4 & 26.4 & 25.1 & 26.2 & 25.9 \\
\hline \multicolumn{13}{|l|}{ Males lines } \\
\hline ICSR29 & 76.8 & 75.0 & 77.5 & 76.4 & 154.1 & 155.8 & 155.1 & 155.0 & 29.3 & 27.1 & 28.1 & 28.2 \\
\hline ICSR53 & 70.5 & 71.8 & 73.3 & 71.9 & 162.5 & 165.9 & 160.4 & 162.9 & 23.9 & 22.8 & 24.4 & 23.7 \\
\hline ICSR89028 & 78.2 & 75.5 & 77.2 & 77.0 & 154.4 & 151.2 & 141.8 & 149.1 & 28.7 & 28.3 & 28.1 & 28.4 \\
\hline ICSR91020 & \begin{tabular}{|l|}
68.3 \\
\end{tabular} & 70.5 & 71.8 & 70.2 & 146.2 & 155.7 & 144.8 & 148.9 & 33.1 & 29.2 & 32.7 & 31.7 \\
\hline ICSR93004 & 78.0 & 78.3 & 78.5 & 78.3 & 143.9 & 145.4 & 141.1 & 143.5 & 24.3 & 23.7 & 26.9 & 25.0 \\
\hline \begin{tabular}{|l|} 
Male's Mean \\
Check varieties \\
\end{tabular} & 74.4 & \begin{tabular}{|l|l|}
74.2 \\
\end{tabular} & 75.7 & 74.7 & 152.2 & 154.8 & 148.6 & 151.9 & 27.9 & 26.2 & 28.0 & 27.4 \\
\hline Hybrid 305 & 70.0 & \begin{tabular}{|l|l|}
69.8 \\
\end{tabular} & 71.7 & 70.5 & 204.2 & 203.4 & 202.2 & \begin{tabular}{|l|}
203.3 \\
\end{tabular} & 31.3 & 29.2 & 30.2 & 30.2 \\
\hline Dorado & 75.0 & \begin{tabular}{|l|}
77.7 \\
\end{tabular} & 75.3 & 76.0 & 131.9 & 132.9 & 126.7 & \begin{tabular}{|l|}
130.5 \\
\end{tabular} & 26.0 & 25.7 & 25.2 & 25.6 \\
\hline Check's Mean & 72.5 & \begin{tabular}{|l|l}
73.8 \\
\end{tabular} & 73.5 & 73.3 & 168.1 & 168.2 & 164.5 & 166.9 & 28.7 & 27.5 & 27.7 & 27.9 \\
\hline Overall Mean & $69.7^{\mathrm{A}}$ & $70.3^{B}$ & $71.7^{\mathrm{C}}$ & 70.6 & $173.3^{A}$ & $172.7^{A}$ & $168.6^{B}$ & 171.5 & $29.7^{\mathrm{A}}$ & $28.0^{\mathrm{B}}$ & $28.5^{\mathrm{C}}$ & 28.7 \\
\hline $\begin{array}{l}\text { Rev. LSD 5\% Genotypes } \\
\text { Rev. LSD 5\% for NPK } \\
\text { level }\end{array}$ & & $\begin{array}{l}0.27 \\
0.50\end{array}$ & & & & $\begin{array}{l}5.86 \\
\text { N.S. }\end{array}$ & & & & $\begin{array}{l}1.46 \\
0.55\end{array}$ & & \\
\hline $\begin{array}{l}\text { Rev.LSD 5\% for NPK } x \\
\text { G }\end{array}$ & & 1.60 & & & & N.S. & & & & 3.78 & & \\
\hline
\end{tabular}

Egypt. J. Agron. 38, No. 2 (2016) 
TABLE 4 (Cont.) Mean performance of the 30 F1 hybrids. their respective parents and check varieties under three NPK levels (100, 75 and 50\%) over both years for biological yield, grain yield and seed index.

\begin{tabular}{|c|c|c|c|c|c|c|c|c|c|c|c|c|}
\hline $\begin{array}{r}\text { Traits } \\
\text { Genotypes }\end{array}$ & \multicolumn{4}{|c|}{ SI; $\mathbf{g}$} & \multicolumn{4}{|c|}{ BY; T/H } & \multicolumn{4}{|c|}{ GY; T/H } \\
\hline $\begin{array}{l}\text { Irrigation } \\
\text { Hybrids }\end{array}$ & $100 \%$ & $75 \%$ & $50 \%$ & Mean & $100 \%$ & $75 \%$ & $50 \%$ & Mean & $100 \%$ & $75 \%$ & $50 \%$ & Mean \\
\hline ICSA20 $\times$ ICSR 29 & 25.6 & 25.9 & 27.6 & 26.4 & 58.4 & 62.8 & 57.8 & 59.7 & 4.1 & 3.8 & 3.8 & 3.9 \\
\hline ICSA20 $\times$ ICSR 53 & 25.8 & 23.6 & 24.5 & 24.6 & 53.6 & 55.8 & 54.9 & 54.8 & 5.9 & 6.9 & 6.0 & 6.3 \\
\hline ICSA20 $\times$ ICSR89028 & 26.4 & 26.7 & 26.0 & 26.4 & 55.7 & 54.3 & 55.8 & 55.3 & 6.0 & 5.6 & 4.7 & 5.4 \\
\hline ICSA20 $\times$ ICSR91020 & 27.3 & 26.0 & 25.1 & 26.1 & 39.6 & 50.4 & 39.4 & 43.1 & 5.8 & 5.2 & 4.1 & 5.0 \\
\hline ICSA20 × ICSR93004 & 25.4 & 25.3 & 24.9 & 25.2 & 58.1 & 62.4 & 58.1 & 59.5 & 5.7 & 5.1 & 4.7 & 5.2 \\
\hline ICSA52 $\times$ ICSR 29 & 29.7 & 29.6 & 26.8 & 28.7 & 46.7 & 42.2 & 40.5 & 43.1 & 6.0 & 3.8 & 4.3 & 4.7 \\
\hline ICSA52 $\times$ ICSR53 & 27.8 & 26.0 & 27.4 & 27.1 & 49.4 & 44.6 & 44.8 & 46.3 & 7.6 & 6.5 & 5.7 & 6.6 \\
\hline ICSA52 $\times$ ICSR 89028 & 31.7 & 28.0 & 24.9 & 28.2 & 54.7 & 38.0 & 43.6 & 45.4 & 5.8 & 5.2 & 5.0 & 5.3 \\
\hline ICSA52 $\times$ ICSR91020 & 27.9 & 26.7 & 27.0 & 27.2 & 32.2 & 34.5 & 25.4 & 30.7 & 6.5 & 5.1 & 4.0 & 5.2 \\
\hline ICSA52 $\times$ ICSR93004 & 29.5 & 26.8 & 26.3 & 27.5 & 48.9 & 53.7 & 47.8 & 50.1 & 7.2 & 7.4 & 6.6 & 7.1 \\
\hline ICSA608 $\times$ ICSR29 & 28.8 & 28.8 & 27.1 & 28.2 & 53.7 & 52.4 & 46.5 & 50.9 & 5.8 & 5.0 & 5.2 & 5.3 \\
\hline ICSA608 $\times$ ICSR 53 & 26.2 & 26.3 & 24.9 & 25.8 & 52.6 & 50.5 & 53.0 & 52.0 & 5.1 & 4.8 & 4.0 & 4.6 \\
\hline \begin{tabular}{|l} 
ICSA608 $\times$ ICSR89028 \\
\end{tabular} & 27.7 & 26.4 & 25.7 & 26.6 & 43.9 & 42.7 & 41.2 & 42.6 & 8.6 & 6.5 & 6.3 & 7.1 \\
\hline \begin{tabular}{|l} 
ICSA608 $\times$ ICSR91020 \\
\end{tabular} & 26.1 & 23.0 & 25.3 & 24.8 & 35.2 & 37.0 & 29.0 & 33.7 & 7.4 & 6.9 & 5.6 & 6.6 \\
\hline \begin{tabular}{|l} 
ICSA608 $\times$ ICSR93004 \\
\end{tabular} & 23.7 & 24.4 & 23.0 & 23.7 & 53.0 & 51.2 & 46.1 & 50.1 & 8.1 & 7.2 & 6.5 & 7.3 \\
\hline ICSA610 $\times$ ICSR 29 & 25.8 & 25.6 & 26.1 & 25.8 & 56.7 & 48.3 & 44.7 & 49.9 & 5.8 & 4.6 & 4.3 & 4.9 \\
\hline ICSA610 $\times$ ICSR53 & 25.9 & 23.8 & 24.9 & 24.9 & 50.2 & 47.5 & 40.0 & 45.9 & 6.5 & 5.8 & 4.6 & 5.6 \\
\hline ICSA610 × ICSR89028 & 26.5 & 24.0 & 24.0 & 24.8 & 49.9 & 45.6 & 40.6 & 45.4 & 7.5 & 7.2 & 5.9 & 6.9 \\
\hline ICSA610 × ICSR91020 & 24.9 & 26.5 & 25.4 & 25.6 & 35.5 & 40.3 & 33.7 & 36.5 & 7.2 & 6.2 & 6.0 & 6.5 \\
\hline ICSA610 × ICSR93004 & 24.9 & 25.6 & 24.1 & 24.9 & 55.5 & 47.0 & 43.8 & 48.8 & 7.9 & 6.5 & 6.2 & 6.9 \\
\hline ICSA613 $\times$ ICSR29 & 30.6 & 26.4 & 28.2 & 28.4 & 49.2 & 59.5 & 51.0 & 53.2 & 6.4 & 4.5 & 3.6 & 4.8 \\
\hline ICSA613 $\times$ ICSR53 & 30.5 & 29.7 & 25.9 & 28.7 & 55.3 & 63.7 & 56.9 & 58.6 & 3.8 & 3.5 & 3.1 & 3.5 \\
\hline ICSA613 × ICSR89028 & 30.9 & 28.7 & 26.7 & 28.8 & 58.5 & 52.4 & 52.3 & 54.4 & 7.5 & 6.6 & 6.6 & 6.9 \\
\hline ICSA613 × ICSR91020 & 30.8 & 28.9 & 26.3 & 28.7 & 46.2 & 45.7 & 33.9 & 41.9 & 6.7 & 3.7 & 3.2 & 4.5 \\
\hline \begin{tabular}{|l|} 
ICSA613 x ICSR93004 \\
\end{tabular} & 26.0 & 25.2 & 23.5 & 24.9 & 46.4 & 45.7 & 42.2 & 44.8 & 5.9 & 5.1 & 4.4 & 5.1 \\
\hline ICSA93 $\times$ ICSR 29 & 28.3 & 30.0 & 28.1 & 28.8 & 56.0 & 56.6 & 50.7 & 54.4 & 4.6 & 4.4 & 3.0 & 4.0 \\
\hline ICSA93 $\times$ ICSR53 & 27.2 & 25.4 & 25.6 & 26.1 & 56.5 & 43.0 & 44.2 & 47.9 & 7.4 & 6.0 & 4.8 & 6.1 \\
\hline ICSA93 × ICSR 89028 & 25.1 & 28.1 & 24.0 & 25.7 & 45.5 & 55.2 & 49.1 & 49.9 & 4.9 & 4.4 & 3.6 & 4.3 \\
\hline ICSA93 $\times$ ICSR91020 & 27.9 & 27.5 & 28.0 & 27.8 & 42.4 & 43.3 & 47.4 & 44.4 & 6.8 & 6.3 & 5.4 & 6.2 \\
\hline ICSA93 $\times$ ICSR93004 & 26.2 & 24.2 & 25.0 & 25.1 & 55.7 & 55.6 & 50.1 & 53.8 & 6.1 & 5.2 & 5.2 & 5.5 \\
\hline \begin{tabular}{|l|} 
Hybrid's mean \\
\end{tabular} & 27.4 & 26.4 & 25.7 & 26.5 & 49.8 & 49.4 & 45.5 & 48.2 & 6.4 & 5.5 & 4.9 & 5.6 \\
\hline \multicolumn{13}{|l|}{\begin{tabular}{|l|} 
Female lines \\
\end{tabular}} \\
\hline \begin{tabular}{|l|} 
ICSB20 \\
\end{tabular} & 24.4 & 22.3 & 24.9 & 23.9 & 12.6 & 14.5 & 20.2 & 15.8 & 6.4 & 5.3 & 4.1 & 5.3 \\
\hline ICSB52 & 29.6 & 29.4 & 27.0 & 28.7 & 16.0 & 8.0 & 15.4 & 13.1 & 5.1 & 4.6 & 3.8 & 4.5 \\
\hline ICSB608 & 24.2 & 23.8 & 22.6 & 23.5 & 16.7 & 14.6 & $\mid 13.2$ & 14.8 & 5.8 & 4.9 & 3.3 & 4.7 \\
\hline ICSB610 & 23.0 & 20.7 & 20.3 & 21.3 & 13.5 & 11.8 & 12.1 & 12.5 & 5.3 & 4.7 & 4.6 & 4.9 \\
\hline ICSB613 & 25.5 & 23.1 & 24.7 & 24.4 & 19.4 & 15.0 & 14.5 & 16.3 & 5.1 & 4.6 & 3.5 & 4.4 \\
\hline ICSB93 & 24.8 & 26.3 & 25.4 & 25.5 & 21.4 & 22.6 & 23.4 & 22.5 & 6.3 & 5.3 & 4.5 & 5.4 \\
\hline Female's Mean & 25.6 & 24.6 & 24.4 & 24.8 & 21.3 & 19.4 & 20.6 & 20.5 & 5.8 & 5.0 & 4.1 & 4.9 \\
\hline \multicolumn{13}{|l|}{ Males lines } \\
\hline ICSR29 & 31.6 & 31.9 & 26.5 & 30.0 & 58.2 & 44.5 & 44.9 & 49.2 & 5.2 & 5.4 & 4.5 & 5.0 \\
\hline ICSR53 & 26.4 & 27.7 & 24.7 & 26.3 & 35.2 & 37.8 & 31.6 & 34.9 & 5.3 & 4.1 & 3.4 & 4.3 \\
\hline ICSR89028 & 26.1 & 27.7 & 28.3 & 27.4 & 39.8 & 39.6 & 31.2 & 36.9 & 6.0 & 5.2 & 4.4 & 5.2 \\
\hline ICSR91020 & 22.0 & 20.1 & 23.9 & 22.0 & 26.4 & 22.5 & 16.7 & 21.9 & 4.9 & 4.1 & 4.1 & 4.4 \\
\hline ICSR93004 & 24.1 & 24.9 & 24.7 & 24.6 & 43.4 & 41.0 & 34.3 & 39.6 & 6.4 & 6.2 & 5.7 & 6.1 \\
\hline Male's Mean & 26.0 & 26.5 & 25.6 & 26.0 & 40.6 & 37.1 & 31.7 & 36.5 & 5.6 & 5.0 & 4.4 & 5.0 \\
\hline \multicolumn{13}{|l|}{ Check varieties } \\
\hline Hybrid 305 & 28.5 & 26.8 & 30.6 & 28.6 & 56.3 & 49.7 & 51.4 & 52.5 & 6.7 & 6.2 & 5.1 & 6.0 \\
\hline Dorado & 27.1 & 26.7 & 24.4 & 26.1 & 28.1 & 28.1 & 25.7 & 27.3 & 6.9 & 6.6 & 6.3 & 6.6 \\
\hline Check's Mean & 27.8 & 26.8 & 27.5 & 27.4 & 42.2 & 38.9 & 38.6 & 39.9 & 6.8 & 6.4 & 5.7 & 6.3 \\
\hline Overall Mean & $26.9^{A}$ & $26.1^{B}$ & $25.6^{\mathrm{C}}$ & 26.2 & $43.8^{A}$ & $42.6^{A}$ & $39.5^{\mathrm{B}}$ & 42.0 & $6.2^{\mathrm{A}}$ & $5.4^{\mathrm{B}}$ & $4.7^{\mathrm{C}}$ & 5.4 \\
\hline Rev. LSD 5\% Genotypes & & 1.73 & & & & 4.86 & & & & 0.35 & & \\
\hline Rev. LSD 5\% for NPK level & & 0.69 & & & & 1.05 & & & & 0.11 & & \\
\hline Rev.LSD 5\% for NPK x G & & 4.34 & & & & N.S. & & & & 0.73 & & \\
\hline
\end{tabular}

Egypt. J. Agron. 38, No. 2 (2016) 
TABLE 5. Heterosis of F1 hybrids over their respective mid parents (MP) and better parents (BP) over NPK levels and years.

\begin{tabular}{|c|c|c|c|c|c|c|c|c|c|c|c|c|}
\hline \multirow{2}{*}{ Hybrids } & \multicolumn{2}{|c|}{$50 \%$ HD } & \multicolumn{2}{|c|}{ PH; cm } & \multicolumn{2}{|c|}{ PL; cm } & \multicolumn{2}{|c|}{ SI; $g$} & \multicolumn{2}{|c|}{ BY; T/H } & \multicolumn{2}{|c|}{ GY; T/H } \\
\hline & MP & $\mathbf{B P}$ & MP & $\mathbf{B P}$ & MP & BP & MP & $\mathbf{B P}$ & MP & BP & MP & $\mathbf{B P}$ \\
\hline ICSA $20 \times \operatorname{ICSR} 29$ & -1.4 & $4.8^{* *}$ & $38.3 * *$ & $28.6 * *$ & 7.9 & 5.1 & -2.1 & -12.1 & $83.7 * *$ & 21.3 & $-24.8 * *$ & $-26.4 * *$ \\
\hline ICSA $20 \times$ ICSR53 & $4.1 * *$ & $7.2 * *$ & $18.4 * *$ & 7.7 & $14.3^{*}$ & 7.9 & -1.8 & -6.3 & $116.4^{* *}$ & $57.1 * *$ & $31.3 * *$ & $19.3^{*}$ \\
\hline ICSA20 $\times$ ICSR 89028 & -0.5 & $6.1^{* *}$ & $18.9 * *$ & $12.6^{*}$ & $12.6^{*}$ & 9.3 & 2.9 & -3.6 & $109.9^{* *}$ & $49.8 * *$ & 3.4 & 3.0 \\
\hline ICSA20 $\times$ ICSR91020 & -1.6 & 0.2 & $25.2 * *$ & $18.7 * *$ & 5.9 & -2.4 & 14.0 & 9.5 & $129.0^{* *}$ & $97.0 * *$ & 5.1 & -3.8 \\
\hline ICSA20 × ICSR 93004 & 2.11 & $10.0^{* *}$ & $29.5 * *$ & $24.9 * *$ & $15.0^{*}$ & 11.2 & 3.9 & 2.4 & $115.3^{* *}$ & $50.6 * *$ & -9.0 & $-15.2 * *$ \\
\hline ICSA $52 \times$ ICSR 29 & $3.3^{*} 1$ & $11.3 * *$ & $22.2 * *$ & $18.4 * *$ & 10.8 & 5.9 & -2.1 & -4.2 & $38.4^{*}$ & -12.3 & -1.1 & -6.3 \\
\hline ICSA5 $2 \times$ ICSR 53 & 1.5 & $6.0 * *$ & $19.9 * *$ & $13.5 * *$ & 6.2 & 2.2 & -1.6 & -5.8 & $92.7 * *$ & 32.7 & $49.9 * *$ & $46.3 * *$ \\
\hline ICSA52 × ICSR 89028 & $-3.2 * *$ & $4.7^{* *}$ & $31.2 * *$ & $29.5 * *$ & $14.2 *$ & 8.6 & 0.6 & -1.8 & $81.7 * *$ & 23.2 & 9.9 & 2.4 \\
\hline ICSA52 × ICSR91020 & -2.6 & 0.4 & $22.8 * * 2$ & $21.4 * *$ & -5.9 & $-14.9 *$ & 7.3 & -5.2 & $75.1^{*}$ & 40.1 & 16.9 & 15.1 \\
\hline ICSA52 × ICSR93004 & $3 * *$ & $4.5^{* *}$ & $18.1 * *$ & $17.3 * *$ & $21.7 * * 2$ & $20.2 * *$ & 3.4 & -4.0 & $90.3^{* *} *$ & 26.8 & $33.3 * *$ & $15.9^{*}$ \\
\hline ICSA608 × ICSR29 & -1.9 & $4.4 * *$ & $42.5 * *$ & $32.0 * *$ & 13.5 & 8.4 & 5.6 & -5.9 & $58.8 * *$ & 3.4 & 10.1 & 6.1 \\
\hline ICSA608 × ICSR53 & 1.2 & $4.3^{* *}$ & $23.5 * *$ & $11.8 *$ & $19.1 * *$ & 14.8 & 3.6 & -1.9 & $109.3^{* *}$ & $49.2 *$ & 2.5 & -1.6 \\
\hline ICSA608 × ICSR89028 & 0.2 & $7.1^{* *}$ & $27.7 * * \mid$ & $20.5^{* *}$ & $17.4 * *$ & 11.5 & 4.6 & -2.7 & $64.7 * *$ & 15.5 & $44.3 * *$ & $36.7 * *$ \\
\hline ICSA608 × ICSR91020 & $-4.9 * *$ & $-3.1 *$ & $22.3 * *$ & $15.5^{* *}$ & 8.8 & -1.6 & 9.0 & 5.5 & $83.7 * *$ & 54.2 & $47.4 * *$ & $42.6 * *$ \\
\hline ICSA608 × ICSR93004 & $4.0^{* * *}$ & $12.1 * *$ & $26.3 * *$ & $21.3 * *$ & $16.0^{*}$ & 14.6 & -1.4 & -3.6 & $84.3 * *$ & 26.7 & $34.5^{* *}$ & $18.8^{*}$ \\
\hline ICSA610 × ICSR 29 & $-7.9 * *$ & -2.4 & $35.0 * *$ & $24.4 * *$ & 7.4 & 4.9 & 0.5 & -13.9 & $62.0 * *$ & 1.5 & -0.5 & -2.2 \\
\hline ICSA610 × ICSR53 & 0.4 & 3.0 & $25.9 * *$ & $13.5 * *$ & 9.2 & 2.8 & 4.4 & -5.4 & $94.0^{* * *}$ & 31.7 & $22.9 *$ & 15.8 \\
\hline ICSA610 × ICSR89028 & -0.2 & $6.2^{* *}$ & $26.0 * *$ & $18.2 * *$ & 2.7 & 0.0 & 1.9 & -9.3 & $84.0 * *$ & 23.1 & $36.4 * *$ & $31.6^{* *}$ \\
\hline ICSA610 × ICSR91020 & $-2.8^{* *}$ & -1.5 & $30.0 * *$ & $22.1 * *$ & 3.8 & -4.1 & $18.2 *$ & 16.5 & $112.5^{* *}$ & $66.7 *$ & $40.2 * *$ & $33.2 * *$ \\
\hline ICSA610 × ICSR93004 & $-5.4 * *$ & 1.5 & $33.4 * *$ & $27.5 * *$ & 5.3 & 1.5 & 8.2 & 1.1 & $87.4 * *$ & 23.2 & $25.4 * *$ & 12.7 \\
\hline ICSA613 × ICSR29 & -1.8 & 3.5 & $30.7 * *$ & $21.2 * *$ & 7.1 & -0.9 & 4.4 & -5.3 & $62.5^{* *}$ & 8.2 & 2.6 & -3.6 \\
\hline ICSA613 × ICSR53 & $-4.8 * *$ & -2.7 & $5 * 9^{* *}$ & $23.3 * *$ & $28.1 * * 2$ & $27.5 * *$ & 13.2 & 9.2 & $129.1 * *$ & $68.2 * *$ & $-20.9^{*}$ & $-22.1^{*}$ \\
\hline ICSA613 × ICSR 89028 & $-3.1 * *$ & 2.6 & $34.2 * *$ & $26.7 * *$ & $15.9 *$ & 6.8 & 11.2 & 5.3 & $104.4^{* *}$ & $47.4 *$ & $43.2 * *$ & 32.3 \\
\hline ICSA613 × ICSR91020 & $-4.0^{* * *}$ & $-3.1 *$ & $36.7 * *$ & $29.2 * *$ & 11.4 & -2.2 & $23.5 * *$ & 17.4 & $119.5^{* *}$ & $91.5 * *$ & 3.5 & 2.8 \\
\hline ICSA613 × ICSR93004 & -1.1 & $5.6^{* *}$ & $22.2 * *$ & $17.6^{* *}$ & $20.4 * *$ & $18.0^{*}$ & 1.6 & 1.3 & $60.3 * *$ & 13.2 & -2.3 & $-15.7 *$ \\
\hline ICSA93 $\times$ ICSR 29 & $2.6^{*}$ & $10.0 * *$ & $17.2 * *$ & $16.9 * *$ & $17.3 * *$ & 13.8 & 3.8 & -4.0 & $51.9 * *$ & 10.7 & $-23.2 * *$ & $-25.5 * *$ \\
\hline ICSA93 × ICSR53 & -1.1 & 2.7 & $14.5 * *$ & $12.1 *$ & 7.9 & 2.3 & 0.7 & -0.9 & $67.0 * *$ & 37.3 & $26.0^{* *}$ & 13.5 \\
\hline ICSA93 $\times$ ICSR89028 & 0.3 & $7.9^{* *}$ & $16.3 * *$ & $13.7 *$ & $14.5^{*}$ & 10.5 & -2.5 & -5.9 & $68.2 * *$ & 35.4 & $-18.8^{*}$ & $-19.8^{*}$ \\
\hline ICSA93 × ICSR91020 & $-4.6 * *$ & -2.2 & 8.2 & 5.8 & 7.5 & -1.4 & $17.1^{*}$ & 9.1 & $99.9 * *$ & $97.3 * *$ & $26.6^{* *}$ & 14.9 \\
\hline ICSA93 × ICSR93004 & 1.3 & $10.0 * *$ & 8.8 & 4.4 & 11.0 & 7.9 & 0.5 & -1.2 & $73.5^{* *}$ & $36.1 *$ & -4.1 & -10.0 \\
\hline
\end{tabular}

*; ** Significant at the 0.05 and 0.01 probability levels, respectively. MP and BP are the mid-parent and better-parent heterosis, respectively .

Egypt. J. Agron. 38, No. 2 (2016) 
TABLE 6. Estimates of general combining ability (GCA) of lines and testers for all studied traits overall environments.

\begin{tabular}{|c|c|c|c|c|c|c|}
\hline Parents & $50 \%$ HD & PH; cm & PL; cm & SI; $\mathbf{g}$ & BY; T/H & GY; T/H \\
\hline \multicolumn{7}{|l|}{ Lines } \\
\hline ICSB20 & $1.36^{* *}$ & -2.01 & 0.37 & $-0.78 * *$ & $6.24 * *$ & $-0.42 * *$ \\
\hline ICSB52 & $-0.65 * *$ & 0.85 & $-0.72 * *$ & $1.22 * *$ & $-5.11 * *$ & $0.20 * *$ \\
\hline ICSB608 & $0.75^{* * *}$ & 0.76 & $0.76^{* *}$ & $-0.69 *$ & $-2.37 * *$ & $0.62 * *$ \\
\hline ICSB610 & $-1.11 * *$ & 2.04 & $-1.00 * *$ & $-1.32 * *$ & $-2.95^{* *}$ & $0.57 * *$ \\
\hline ICSB613 & $-0.61 * *$ & $6.03 * *$ & 0.19 & $1.37 * *$ & $2.35^{* *}$ & $-0.60 * *$ \\
\hline ICSB93 & 0.25 & $-7.67 * *$ & 0.40 & 0.19 & $1.84 *$ & $-0.38 * *$ \\
\hline S.E. (gi) lines & 0.140 & 1.093 & 0.260 & 0.833 & 0.893 & 0.065 \\
\hline S. E. (gi-gj) & 0.198 & 1.546 & 0.368 & 1.178 & 1.263 & 0.092 \\
\hline \multicolumn{7}{|l|}{ Testers } \\
\hline ICSR29 & $0.82^{* * *}$ & $10.00^{* *}$ & 0.25 & $1.22 * *$ & $3.65^{* *}$ & $-0.96^{* *}$ \\
\hline ICSR53 & $-0.44 * *$ & $3.43 * *$ & $-1.38 * *$ & -0.34 & $2.67 * *$ & $-0.14 * *$ \\
\hline ICSR89028 & $1.17 * *$ & -1.13 & $0.96^{* *}$ & 0.23 & 0.60 & $0.42^{* *}$ \\
\hline ICSR91020 & $-3.76 * *$ & $-3.63^{* *}$ & $0.63^{* *}$ & 0.18 & $-9.85 * *$ & 0.10 \\
\hline ICSR93004 & $2.20 * *$ & $-8.66^{* *}$ & -0.46 & $-1.29 * *$ & $2.94 * *$ & $0.58 * *$ \\
\hline S.E. (gi) testers & 0.128 & 0.998 & 0.237 & 0.760 & 0.815 & 0.059 \\
\hline S.E. (gi-gj) & 0.180 & 1.411 & 0.336 & 1.075 & 1.153 & 0.084 \\
\hline
\end{tabular}

*; ** Significant at the 0.05 and 0.01 probability levels, respectively.

\section{Panicle length (PL)}

Data exhibited in Table 4 show that panicle length was affected significantly by the reduction in NPK levels. The large panicle in the hybrid is initiated earlier and develops faster than in its parents (Blum, 1990). However, the majority of the crosses had longer panicle than their parents indicating the existence of midparent (MP) and better-parent heterosis (BP) (Table 5). About half of the crosses had longer panicle than the best check variety (Hybrid 305). The cross ICSA93 $\times$ ICSR29 had longer panicle $(32.0 \mathrm{~cm})$ followed by ICSA608 $\times$ ICSR89028 which recorded $31.7 \mathrm{~cm}$. The testers had longer panicle than lines. For combining ability effects, the highest positive GCA of 0.76 and 0.96 were manifested by the line ICSB608 and tester ICSR89028, respectively (Table 6). Implying that both the parents were good general combiners. For SCA effects, half crosses showed positive SCA effects, hence, the cross (ICSA52 $\times$ ICSR93004) had positive and highly significant SCA effects $(2.34 * *)$ followed by the cross (ICSA613 $\times$ ICSR53) $\left(2.07^{* *}\right)$. The previous results indicated that these crosses can be considered as good combiners for panicle length. This is in agreement with the findings of Hovny \& El-Dsouky (2007), Abdel-Mottaleb (2009), Essa (2009), Mahdy et al. (2011) and Amir \& Mohamed (2015). 
TABLE 7. Estimates of specific combining ability (SCA) of $30 F_{1}$ hybrids for all studied traits overall environments.

\begin{tabular}{|c|c|c|c|c|c|c|}
\hline Hybrids & $50 \%$ HD & PH; cm & PL; cm & SI; g & BY; T/H & GY; T/H \\
\hline ICSA20 $\times$ ICSR29 & $-1.39 * *$ & $9.56 * *$ & -0.67 & -0.57 & 1.56 & $-0.33^{*}$ \\
\hline ICSA20 $\times$ ICSR53 & $1.48 * *$ & $-7.72 * *$ & 0.19 & -0.78 & -2.38 & $1.26^{* *}$ \\
\hline ICSA20 × ICSR 89028 & $-0.85^{* *}$ & $-10.68^{* *}$ & 0.05 & 0.39 & 0.20 & -0.16 \\
\hline ICSA20 × ICSR91020 & 0.03 & 0.64 & 0.28 & 0.21 & -1.50 & -0.20 \\
\hline ICSA20 × ICSR93004 & $0.73 *$ & $8.20 * *$ & 0.16 & 0.74 & 2.12 & $-0.57 * *$ \\
\hline ICSA52 $\times$ ICSR 29 & $3.12 * *$ & $-9.08 * *$ & 0.63 & -0.22 & -3.64 & -0.10 \\
\hline $\mathrm{ICSA} 52 \times \mathrm{ICSR} 53$ & $0.83 * *$ & -1.12 & -1.33 & -0.36 & 0.46 & $0.95 * *$ \\
\hline ICSA52 × ICSR89028 & $-1.62 * *$ & $11.74 * *$ & 0.96 & 0.21 & 1.72 & $-0.85^{* *}$ \\
\hline ICSA52 × ICSR91020 & 0.48 & 1.81 & $-2.60^{* *}$ & -0.73 & -2.60 & $-0.70 * *$ \\
\hline ICSA52 × ICSR93004 & $-2.81 * *$ & -3.35 & $2.34 * *$ & 1.10 & 4.05 & $0.70 * *$ \\
\hline ICSA608 $\times$ ICSR29 & $-1.17 * *$ & $12.06 * *$ & -0.15 & 1.20 & 1.34 & 0.11 \\
\hline$. \mathrm{ICSA} 608 \times$ ICSR53 & -0.02 & -3.72 & 0.34 & 0.30 & 3.48 & $-1.46^{* *}$ \\
\hline ICSA608 × ICSR89028 & 0.26 & -1.65 & 0.31 & 0.54 & -3.86 & $0.53 * *$ \\
\hline ICSA608 × ICSR91020 & $-1.69 * *$ & $-6.93 * *$ & 0.13 & -1.21 & -2.27 & $0.36^{* *}$ \\
\hline ICSA608 × ICSR93004 & $2.62 * *$ & 0.24 & -0.62 & -0.84 & 1.30 & $0.46^{* * *}$ \\
\hline ICSA610 $\times$ ICSR 29 & $-3.42 * *$ & -0.92 & 0.65 & -0.59 & 1.00 & -0.27 \\
\hline ICSA610 × ICSR53 & $1.56 * *$ & -2.25 & 0.35 & 0.01 & -2.06 & $-0.39 * *$ \\
\hline ICSA610 × ICSR89028 & $2.11 * *$ & $-6.28 * *$ & $-1.21 *$ & -0.62 & -0.50 & $0.31 *$ \\
\hline ICSA610 × ICSR91020 & $1.83 * *$ & 1.68 & 1.11 & 0.24 & 1.06 & 0.21 \\
\hline ICSA610 × ICSR93004 & $-2.08 * *$ & 7.77 & -0.90 & 0.95 & 0.50 & 0.13 \\
\hline ICSA613 × ICSR29 & $0.80 * *$ & $-9.88 * *$ & $-2.18 * *$ & -0.70 & -0.99 & $0.84 * *$ \\
\hline ICSA613 × ICSR53 & $-2.27 * *$ & $9.66 * *$ & $2.07 * *$ & 1.14 & $5.37 * *$ & $-1.39 * *$ \\
\hline ICSA613 × ICSR89028 & -0.22 & 2.37 & -0.48 & 0.66 & 3.20 & $1.51 * *$ \\
\hline ICSA613 × ICSR91020 & $0.83 * *$ & $8.22 * *$ & 0.52 & 0.59 & 1.19 & $-0.53 * *$ \\
\hline ICSA613 × ICSR93004 & $0.86 * *$ & $-10.38 * *$ & 0.07 & $-1.70 * *$ & $-8.76^{* *}$ & $-0.43 * *$ \\
\hline ICSA93 × ICSR29 & $2.06 * *$ & -1.75 & $1.73 * *$ & 0.88 & 0.72 & -0.25 \\
\hline ICSA93 × ICSR53 & $-1.57 * *$ & $5.16^{*}$ & -1.62 & -0.32 & $-4.88 *$ & $1.02 * *$ \\
\hline ICSA93 × ICSR 89028 & 0.31 & 4.50 & 0.38 & -1.19 & -0.75 & $-1.33 * *$ \\
\hline ICSA93 × ICSR91020 & $-1.47 * *$ & $-5.42 *$ & 0.56 & 0.89 & $4.13 *$ & $0.85 * *$ \\
\hline ICSA93 × ICSR93004 & $0.68 *$ & -2.49 & -1.05 & -0.26 & 0.78 & $-0.30^{*}$ \\
\hline S.E. SCA & 0.312 & 2.444 & 0.581 & 0.647 & 1.997 & 0.145 \\
\hline SE (Sij-Skl) & 0.442 & 3.457 & 0.822 & 0.915 & 2.824 & 0.205 \\
\hline
\end{tabular}

*; ** Significant at the 0.05 and 0.01 probability levels, respectively.

Egypt. J. Agron. 38, No. 2 (2016) 


\section{Seed Index $(g)$}

Presented data in Table 4 investigate that seed index was decreased upon decreasing NPK fertilizer so that, L2 and L3 caused 2.97 and 4.83\% reduction in seed index, respectively. This reduction was not significant. Similar results were obtained by Omar et al. (2014), who stated that plant height, 1000-grain weight and grain yield/plant of hybrids and parents were decreased with increasing nitrogen stress. Five crosses out of thirty gave higher seed index than the best check variety (Hybrid 305). However, the mean performance of F1 hybrids per se showed that hybrid ICSA613 $\times$ ICSR91020 manifested highest $(28.7 \mathrm{~g})$ seed index and recorded the highest positive and significant mid-parent heterosis $(23.5 \%)$ while next maximum value $(28.7 \mathrm{~g})$ was given by ICSA93 $\times$ ICSR29 (Table 5). Among the lines, ICSB52 (28.68 g) and testers, ICSR29 (29.99 g) recorded maximum seed index (Table 4). Among the parental lines, ICSB613 $(1.37 * *), \operatorname{ICSB} 52(1.22 * *)$ and tester, ICSR29 $(1.22 * *)$ exhibited positive and highly significant GCA effects (Table 6). The SCA effects revealed that sixteen out of thirty F1 hybrids exhibited positive non-significant effects, yet the maximum SCA effect (1.20) was given by ICSA608 $\times$ ICSR29 and next ranker was ICSA613 $\times$ ICSR53. These results are in accordance with the findings of Hovny \& El-Dsouky (2007), Abdel-Mottaleb (2009), Essa (2009), Makanda $e t$ al. (2010), Mahdy et al. (2011) and Aminu \& Izge (2013).

\section{Biological yield $\left(t h a^{-1}\right)$}

Biological yield was decreased significantly as NPK fertilizer decreased so that NPK level 2 and NPK level 3 caused 2.7 and 9.7\% reduction in biological yield $\left(\mathrm{t} \mathrm{ha}^{-1}\right)$, respectively as compared with level 1 . This is to be logic since the same trend was true with regard to plant height, panicle length and seed index. Abou-Amer \& Kewan (2014) stated that fodder yield was increased significantly by increasing $\mathrm{N}$ and $\mathrm{P}$ fertilizer levels. Also, Hussein \& Alva (2014) reported that the increased rates of N, P, K increased the plant growth and biomass. Sorghum hybrids produce more biomass as compared with their parents (Sahoo, 2010). On average, most crosses exhibited remarkable increase in biological yield than their parents and the check varieties indicating the existence of heterosis under all NPK levels. Nine crosses out of thirty produced higher biological yield than the best check variety (Hybrid 305). However, the highest cross in biological yield was ICSA20 $\times$ ICSR29 and recorded $\left(59.7 \mathrm{t} \mathrm{ha}^{-1}\right)$ followed by the cross ICSA20 $\times$ ICSR93004 and recorded $\left(59.5 \mathrm{t} \mathrm{ha}^{-1}\right)$. However, all crosses showed positive and high significant mid-parent heterosis, whereas few crosses showed significant high-parent heterosis (Table 5). The cross (ICSA613 $\times$ ICSR53) showed significant and positive mid-parent (129.1 $\%)$ and high-parent $(68.2 \%)$ heterosis followed by the cross ICSA20 $\times$ ICSR91020 and recorded 129 and 97\% mid- and high parent heterosis, respectively. For combining ability effects, the highest positive GCA of $6.24 * *$ and $3.65^{* *}$ were manifested by the line ICSB20 and tester ICSR29, respectively (Table 6). implying that both the parents were good general combiners. Concerning SCA effects, few crosses showed positive SCA effects, hence, the cross ICSA613 $\times$ ICSR53 positive and highly significant SCA effects $\left(5.37^{* *}\right)$ followed by the cross ICSA93 $\times$ ICSR91020 $(4.13 *)($ Table 7$)$. The previous 
results indicated that these crosses can be considered as good combiners for biological yield. Haussmann et al. (1999) studied the quantitative genetic parameters of grain sorghum under variable stress conditions. They found that the relative hybrid mean superiority over the mid parent values was highest for grain yield followed by plant height and above ground dry matter. Our findings are in accordance with the findings of Hovny \& El-Dsouky (2007), AbdelMottaleb (2009), Essa (2009), Mahdy et al. (2011), Omar et al. (2014) and Amir \& Mohamed (2015).

Grain yield $\left(t h a^{-1}\right)$

Grain yield occupies a unique place among plant characters. As an average, grain yield $\left(\mathrm{t} \mathrm{ha}^{-1}\right)$ of sorghum genotypes, decreased as a result of reducing the amount of NPK fertilizers as compared with recommended NPK fertilizers. Average reduction in grain yield was 12.9 and $24.2 \%$ in $75 \%$ and $50 \%$ NPK, respectively. This is to be expected since the same obtained was observed with regard to seed index and biological yield and consequently produced the highest mean values of grain yield. Shrotriya (1998) reported that balanced application of NPK caused up to $122 \%$ increase in sorghum yield in India. El-Aref et al. (2005) reported that nitrogen levels significantly affected the grain yield and stated that applying $125 \mathrm{~kg} \mathrm{~N} / \mathrm{fad}$ was more effective compared with other studied nitrogen levels. Al-Nagar et al. (2006) reported that Low-N stress caused a significant reduction in grain yield / plant of 17.9 and $15.2 \%$ for parental lines and their F1s, respectively. Also, significant increase in grain yield by increasing NP levels were observed by Abou-Amer \& Kewan (2014). Dorado recorded higher grain yield than Hybrid 305 cultivar, this result is in contrast with those obtained by Abdo et al. (2014) under water stress conditions. Among the F1 hybrids per se, the crosses ICSA608 × ICSR93004 and ICSA52 × ICSR93004 produced top $\left(7.2\right.$ ton $\left.\mathrm{ha}^{-1}\right)$ and next maximum $\left(7.1\right.$ ton ha $\left.{ }^{-1}\right)$ grain yield (Table 4). Seven crosses out of thirty produced higher grain yield than the best check variety (Drorado). Among the parents, tester ICSR93004 produced highest grain yield $\left(6.1\right.$ ton $\left.\mathrm{ha}^{-1}\right)$ whereas among the females, maximum grain yield $\left(5.4 \mathrm{t} \mathrm{ha}^{-1}\right)$ was yielded by the line ICSB93 (Table 4). This result indicated that these high yielding hybrids and parental lines are tolerant to low NPK fertility. It was observed that the tester ICSR93004 has participated the highest yielding crosses. In plant breeding, it is normally assumed that when good performing parents are crossed with each other, they are anticipated to produce better hybrids but this assumption was not always true (Baloch \& Bhutto, 2003). The majority of the crosses showed positive heterosis in relation to their parents, thirteen crosses had positive and high significant mid- and high parent heterosis (Table 5). The cross $($ ICSA52 $\times$ ICSR53) showed significant and positive mid-parent $(49.9 \%)$ and high-parent $(46.3 \%)$ heterosis followed by the cross ICSA608 $\times$ ICSR91020 and recorded 47.4 and $42.6 \%$ mid- and high parent heterosis, respectively. By pooling the review of literature on heterosis studies, Abo-Elwafa (2005), Hovny \& El-Dsouky (2007) and Abdel-Mottaleb (2009) observed high relative heterosis and heterobeltiosis for grain yield. For GCA effects, the female line ICSB608 showed the highest positive GCA effects $(0.62 * *)$ whereas the male line ICSR93004 gave maximum (0.58**) GCA effects. For SCA, effects, the crosses Egypt. J. Agron. 38, No. 2 (2016) 
ICSA613 $\times$ ICSR89028 and ICSA20 $\times$ ICSR53 gave positive and highly significant SCA effects indicating that these crosses can be considered desirable combiners. This is in agreement with the findings of Hovny \& El-Dsouky (2007), Abdel-Mottaleb (2009), Essa (2009), Mahdy et al. (2011), Omar et al. (2014) and Amir \& Mohamed (2015).

\section{Correlation among studied traits}

Data in Table 8 show the mutual correlation among traits studied overall NPK levels and over two seasons. Looking at the correlation data under NPK levels, it can be concluded that, heading dates was correlated positively and significantly with each of BY $\left(\mathrm{r}=0.252^{* *}\right)$ and SI $\left(\mathrm{r}=0.090^{*}\right)$, whereas, it was negative but non-significant with GY and that may be due to the decline of the amount of NPK fertilizers added as stress conditions. This decline led to decrease GY and delayed heading time. Plant height was associated positively and significantly with all studied traits with exception of days to $50 \%$ heading. Biological yield was correlated positively and significantly with GY and SI. Also, data showed that there was a positive correlation but weak between GY and SI and this may be due to the weakness of NPK accumulation as a result of fertilizers deficiency. Tag El-Din et al. (2012) found a positive and nonsignificant correlation between grain yield and 1000-kernel weight. Also, Almeida Filho et al. (2014) reported a positive association between plant height and grain yield and negative correlation with days to flowering. Omar et al. (2014) found that plant height had positive and highly significant correlation with grain yield/plant under two $\mathrm{N}$ levels. While, 1000 grain weight had negative and highly significant correlation with grain yield / plant under two $\mathrm{N}$ levels.

TABLE 8. Phenotypic correlations among six traits computed overall environments.

\begin{tabular}{|l|c|c|c|c|c|}
\hline \multicolumn{1}{|c|}{ Traits } & PH; cm & PL; cm & SI; $\mathbf{g}$ & BY; T/H & GY; T/H \\
\hline $50 \%$ HD & 0.065 & -0.012 & $0.090^{*}$ & $0.252^{* *}$ & -0.034 \\
\hline PH; cm & & $0.349^{* *}$ & $0.313^{* *}$ & $0.594^{* *}$ & $0.183^{* *}$ \\
\hline PL; cm & & & $0.133^{* *}$ & $0.301 * *$ & 0.024 \\
\hline SI; g & & & & $0.267 * *$ & 0.061 \\
\hline BY; T/H & & & & & $0.088^{*}$ \\
\hline
\end{tabular}

*; ** Significant at the 0.05 and 0.01 probability levels, respectively.

\section{Genetic components}

Data in Table 9 show the genetic components and contribution of the lines, testers and their interaction of all studied traits overall NPK levels and years. The line $\times$ tester analysis revealed that the contribution of the testers to the total sum of squares was higher than of lines in all studied traits except in SI. Since the maximum contribution $(55.26 \%)$ of the testers was noted for days to $50 \%$ heading while the lowest values was recorded for grain yield $(26.89 \%)$. Lines were contributed as maximum value $(45.07 \%)$ in seed index and the lowest one was observed for days to $50 \%$ heading $(9.73 \%)$. For the line $\times$ tester interaction, 
the maximum contribution to the total some of squares was $52.91 \%$ in panicle length whereas the minimum contribution was $17.68 \%$ in case of biological yield. It was observed that the variance due testers was higher than those of lines and that confirmed by the contribution of lines and testers in previous discussion. The results revealed that, dominance variance $\left(\sigma_{D}^{2}\right)$ was high and additive genetic variance $\left(\sigma_{A}^{2}\right)$ was low in magnitude for all the traits. The ratio of $\left(\sigma_{A}^{2} / \sigma_{D}^{2}\right)$ ranged from 0.05 (panicle length and grain yield) to 0.55 (biological yield). The observations on portioning of combining ability variance into additive variance $\left(\sigma_{A}^{2}\right)$ and dominance variance $\left(\sigma_{D}^{2}\right)$ indicated role of both additive and dominance gene action. The magnitude of non-additive variance was higher than the additive variance by many folds for all studied traits. Broadsense heritability was moderate to high $(43.21-85.74 \%)$ for panicle length and days to $50 \%$ heading but low for seed index $(31.46 \%)$. Whereas narrow-sense heritability was very low and ranged between $0.69 \%$ (panicle length) and $4.18 \%$ (days to 50\% heading). Mohammed (2009) reported that additive is important in the expression of days to flower, forage yield and stem diameter and nonadditive gene actions is important in the expression of plant height. Mahdy et al. (2011) found that both additive and non-additive are important for the inheritance of plant height and grain yield, while they found that the additive effect is controlling days to $50 \%$ flowering. Several researchers have indicated that additive and non-additive are important in the inheritance of grain yield and some agronomic traits (Kenga et al., 2004; Abdel-Mottaleb, 2009, Mohammed, 2009 and Mahdy et al., 2011).

In conclusion, significant differences were observed among entries, parents, parents vs. crosses, crosses, lines, testers, lines $\times$ testers, turned for all the characters studied suggesting that the experimental materials possessed considerable variability. The majority of the crosses were earlier, taller, longer panicle, higher in biological yield, higher in grain yield and heavier in seed index than their parents under combined NPK levels and two seasons. In addition, decreasing NPK fertilizers decline in plant height, panicle length, biological yield, seed index and grain yield/plant. While, decreasing NPK fertilizers led to increasing days to $50 \%$ flowering. These results are in harmony with those obtained by Abo-Elwafa (2005), Hovny \& El-Dsouky (2007), Abdel-Mottaleb (2009) and Omar et al. (2014). They concluded that most of the F1 crosses were earlier, taller, heavier grain weight and higher grain yield compared with their parents. A comparison of GCA effects of individual lines for grain yield showed that much of the positive GCA obtained was contributed by ICSA608. ICSA610, ICSR89028 and ICSR93004 under NPK levels and over two years. This suggests a wide adaptation and high potential of these lines for use as a parent in developing well-adapted hybrids with high yield potential and can be considered as best general combiners. On the other hand, the female line ICSB610 showed significant and negative GCA effects for days to $50 \%$ heading and panicle length

Egypt. J. Agron. 38, No. 2 (2016) 
and positive GCA effects for grain yield and plant height. It may be used to develop high yielding, early flowering, and tall hybrids with short panicle length. For SCA, effects, the crosses ICSA613 × ICSR89028 and ICSA20 × ICSR53 gave positive and highly significant SCA effects indicating that these crosses can be considered desirable combiners. The crosses which recorded high SCA effects, had high yield per se and one of the parents involved with highest GCA effects. Thus, hybrid combination with high mean, with favorable SCA estimate and involving at least one of the parents with high GCA, would tend to increase the concentration of favorable alleles; an appreciable situation to any breeder.

TABLE 9. Genetic components estimates and proportional contribution to the total variation of the lines $\times$ tester analysis for the studied traits over environments.

\begin{tabular}{|l|c|c|c|c|c|c|}
\hline & $\mathbf{5 0 \%}$ HD & PH; cm & PL; $\mathbf{c m}$ & SI; $g$ & BY; T/H & GY; T/H \\
\hline $\begin{array}{l}\text { Genetic components } \\
\text { estimates }\end{array}$ \\
\hline Cov H.S. Lines & 0.09 & 7.50 & 0.11 & 1.07 & 15.06 & 0.12 \\
\hline Cov H.S.Tester & 4.64 & 39.13 & 0.56 & 0.69 & 29.47 & 0.23 \\
\hline Cov.H.S. (average) & 0.26 & 2.55 & 0.04 & 0.10 & 2.46 & 0.02 \\
\hline Cov F.S. (Hybrids) & 13.29 & 151.30 & 2.83 & 3.61 & 92.25 & 1.47 \\
\hline Additive var. $\left(\sigma^{2} \mathrm{~A}\right)$ & 0.51 & 5.11 & 0.07 & 0.20 & 4.91 & 0.04 \\
\hline Dominance var. $\left(\sigma^{2} \mathrm{D}\right)$ & 3.94 & 61.26 & 1.53 & 0.48 & 8.90 & 0.82 \\
\hline$\sigma^{2} \mathrm{~A} / \sigma^{2} \mathrm{D}$ & 0.13 & 0.08 & 0.05 & 0.41 & 0.55 & 0.05 \\
\hline$\left(\sigma^{2} \mathrm{D} / \sigma^{2} \mathrm{~A}\right)^{0.5}$ & 2.77 & 3.46 & 4.53 & 1.56 & 1.35 & 4.65 \\
\hline$H^{2}{ }_{B}$ & 85.74 & 77.95 & 43.21 & 31.46 & 71.43 & 70.62 \\
\hline$H^{2}{ }_{N}$ & 4.18 & 1.05 & 0.69 & 1.79 & 1.96 & 2.79 \\
\hline $\begin{array}{l}\text { Proportional contribution } \\
\text { to total variation }(\%)\end{array}$ & \multicolumn{7}{|l|}{} \\
\hline Lines & 9.73 & 17.02 & 17.26 & 45.07 & 30.25 & 21.71 \\
\hline Testers & 55.26 & 39.27 & 29.82 & 29.06 & 52.06 & 26.89 \\
\hline LxT & 35.01 & 43.71 & 52.91 & 25.86 & 17.68 & 51.40 \\
\hline
\end{tabular}

\section{References}

Abdel-Mottaleb, A.A. (2009) Heterosis and combining ability in grain sorghum (Sorghum bicolor L. Moench) under optimum and low level of nitrogen. Ph.D. Thesis, Faculty of Agriculture, Assiut University, Egypt.

Abdo, F.A., Madkour, M.A., El-Batal, M.A. and Anton, N.A. (2014) Physiological behavior of two grain sorghum genotypes under different irrigation of water applied levels. Research Journal of Agriculture and Biological Sciences, 10(2), 154-161, 2014. 
Abo-Elwafa, A., Ahmed, T.A., Hassaballa, E.A. and Sayed, M.A. (2005) Heterosis and line $\mathrm{x}$ tester analysis of combining ability in grain sorghum (Sorghum bicolor $\mathrm{L}$. Moench). Assiut Journal of Agricultural Science, 36 (1),159-175.

Abou-Amer, A. and Kewan, K. (2014) Effect of NP fertilization levels on sorghum (Sorghum bicolor L.) yield and fodder quality for animals. Alex. J. Agric. Res. 59, 5159.

Ali, M.A., Abbas, A., Niaz, S., Zulkiffal, M. and Ali, S. (2009) Morpho-physiological criteria for drought tolerance in sorghum (Sorghum bicolor L. Moench) at seedling and post-anthesis stages. Int. J. Agric. Biol. 11, 674-680.

Almeida Filho, G.E., Tardin, F.D., Vilela de Resende, M.D., Silva, F.F., Granato, I.S.C. and de Menezes, C.B. (2014) Genetic evaluation of grain sorghum hybrids in Brazilian environments using the REML/BLUP procedure. Sci. agric. 71 (2).

Al-Nagar, A.M., El-Kadi, D.A. and Abo-Zaid, Zeinab S.H. (2006) Quantitative genetic parameters of grain sorghum traits contributing to Low-N tolerance. Egypt J. Plant Breed, 10, 79-102.

Aminu, D. and Izge, A. U. (2013) Gene action and heterosis for yield and yield traits in maize (Zea mays L.), under drought conditions in Northern Guinea and Sudan savannas of Borno State, Nigeria Peak Journal of Agricultural Sciences , 1 (1),17-23.

Amir, A.A. and Mohamed, E.I. (2015) Inheritance of sorghum yield and its components under low nitrogen fertilizer using line by tester analysis. Egypt. J. Plant Breed. 19(4), $1117-1131$

Baloch, M.J. and Bhutto, H.U. (2003) Design-II analysis for estimating general and specific combining ability effects of cotton leaf curl virus resistant inbreed parents. Zagazig J. Agric. Res. 30, 635-649.

Bantilan, M.C.S., Deb, U.K., Gowda, C.L.L., Reddy, B.V.S., Obilama, A.B. and Evenson, R.E. (2004) Sorghum genetic enhancement: Research process, dissemination and impacts. ICRISAT. 201- 221.

Bibi, A., Sadaqat, H.A., Akram, H.M. and Mohammed, M.I. (2010) Physiological markers for screening sorghum (Sorghum bicolor) germplasm under water stress condition. Intl. J. Agric. Biol., 12, 451-455.

Blum, A., Ramaiah, S., Kanemasu, E. T. and Paulsen, G. M. (1990) The Physiology of heterosis in sorghum with respect to environmental stress. Annals of Botany, 65, 149-158.

Cruz, C.D. and Regazzi, A.J. (1994) "Modelos Biometricos Aplicados ao Melhoramento Genetico”. Universidade Federal de Vic,osa,Imprensa Universitaria, Vic „osa, Minas Gerais, Brazil.

Ejeta, G. and Knoll, J.E. (2007) Marker-assisted selection in sorghum. In: "GenomicAssisted Crop Improvement”: Varshney, R.K., and Tuberosa, R. (Ed.) pp: 187-205 Vol. 2: Genomics Applications in Crops Springer Publications. The Netherlands.

Egypt. J. Agron. 38, No. 2 (2016) 
El-Aref, Kh. A. O., Abdel-Mawly, S. E. and Abo-Elhamd, A. S. (2005) Improving yield and water use efficiencies of two sorghum cultivars irrigated by surface and drip irrigation systems and fertilized by nitrogen. Ass. Univ. Bull. Environ. Res. 8(2),67-80.

Essa, H.M.H. (2009) Breeding grain sorghum for drought tolerance M.Sc. Thesis, Faculty of Agriculture, Ain Shams University, Egypt.

Fageria, N. K., Baligar, V. C. and Jones, C. A. (2011) "Growth and Mineral Nutrition of Field Crops". Boca Raton, FL: CRC Press.

Faiz, F. A. Sabar, M., Awan, T.H., Tjaz, M. and Manzoor, Z. (2006) Heterosis and combining ability analysis in basmati rice hybrids. J. Anim. PI. Sci. 16 (1 - 2), 56-59.

Falconer, D.S. (1989) “Introduction to Quantitative Genetic", $3^{\text {rd }}$ ed. Longman, Essex, UK, pp. 275-276.

FAOSTAT(2012) http://faostat.fao.org. Accessed on 20/5/2012.

FAOSTAT (2014) http://faostat3.fao.org/browse/Q/QC/E. Accessed on May 12, 2016.

Haussmann, B.I.G., Obilana, A.B., Ayiecho, P.O., Blum, A., Schipprack, W. and Geiger, H.H. (1999) Quantitative genetic parameters of sorghum [Sorghum bicolor (L.) Moench] grown in semi-arid areas of Kenya. Euphytica, 105, 109-118.

Hochholdinger, F. and Hoecker, N. (2007) Towards the molecular basis of heterosis. Trends Plant Sci. 12, 427-432.

House, L.R., Verma, B.N., Ejeta, G., Rana, B.S., Kapran, I., Obilana, A.B. and Reddy, B.V. (1997) Developing countries breeding and potential of hybrid sorghum. In: Proceedings of the International Conference on Genetic Improvement of Sorghum and Pearl Millet, Lubbock, Texas, USA pp 84-96.

Hovny, M.R.A. and El-Dsouky, M.M. (2007) performance of some grain sorghum lines and their hybrids under optimum and low input nitrogen conditions. Assiut J. Agric. Sci. 39, 67-90.

Hussein, M.M. and Alva, A.K. (2014) Growth, yield and water use effeciency of forage sorghum as affected by NPK fertilizer and deficit irrigation. American Journal of Plant Sciences, 5, 2134-2140. http://dx.doi.org/10.4236/ajps.2014.513225.

Kanbar, O. Z., Kanbar, A. and Shehab, S. (2011) Combining ability and heterosis for some yield traits in sorghum (Sorghum bicolor L. Moench) using (linextester) design J. Plant Production, Mansoura Univ. 2 (8), $1009-1016$.

Kempthorne, O. (1957) “An Introduction to Genetic Statistics”. John Wiley and Sons, inc New York. P. 545.

Kenga, R., Alabi, S.O. and Gupta, S.C. (2004) Combining ability studies in tropical sorghum (Sorghum bicolor L. Moench). Field Crops Res. 88, 251-260.

Lamkey, K.R. and Edwards, J. W. (1999) The quantitative Genetics of heterosis. In: J.G. Coors and S. Pandey (Ed.) Proceedings of the International Symposium on the 
Genetics and Exploitation of Heterosis in Crops, CIMMYT, Mexico City, Mexico, 17-22 Aug. 1997. ASA, CSSA, and SSSA, Madison, WI pp. 31-48.

Mahdy, E.E., Ali, M.A. and Mahmoud, A.M. (2011) The effect of environment on combining ability and heterosis in grain sorghum (Sorghum bicolor L. Moench). Asian J. Crop Sci. 3(1),1-15.

Makanda, I., Tongoona, P., Derera, J., Sibiya, J. and Fato, P. (2010) Combining ability and cultivar superiority of sorghum germplasm for grain yield across tropical low- and mid-altitude environments. Field Crops Research, 116, 75-85.

Mohammed, M. (2009) Line x tester analysis across locations and years in Sudanese x exotic lines of forage sorghum. Journal of Plant Breeding and Crop Science. 1(9), 311-319.

Mueller, N. D., Gerber, J. S., Johnston, M., Ray, D. K., Ramankutty, N. and Foley, J. A. (2012) Closing yield gaps through nutrient and water management. Nature, 490, 254-257.

Omar, K.A., Mahmoud, K.M. and Mohamed, M.E. (2014) Heterosis in grain sorghum under two levels of nitrogen fertilizer. Middle East Journal of Agriculture Research, 3(2), 176-185.

Rashid, M., Cheema, A. A. and Ashraf, M. (2007) Line x tester analysis in basmati rice. Pak. J. Bot. 39 (6), 2035-2042.

Ringo, J., Onkware, A., Mgonja, Mary, Deshpande, S., Rathore, A., Mneney, E. and Gudu, E. (2015) Heterosis for yield and its components in sorghum (Sorghum bicolor L. Moench) hybrids in dry lands and sub-humid environments of East Africa. AJCS 9(1), 9-13.

Rooney, W.L., Blumenthal, J., Bean, B. and Mullet, J.E. (2007) Designing sorghum as a dedicated bioenergy feedstock. Biofuel Bioprod. Bioref. 1, 147-157.

Sahoo, L., Schmidt, J.J., Pedersen, J.F., Lee, D. J. and Lindquist, J. L. (2010) Growth and fitness components of wild x cultivated Sorghum bicolor (Poaceae) hybrids in Nebraska. American Journal of Botany, 97(10), 1610-1617.

Saleem, M. Y., Mirza, J. I. and Haq, M. A. (2008) Heritability, genetic advance and heterosis. 2002. In line x tester crosses of basmati rice. J. Agric. Res. 46 (1), 15-27.

Sarker, U., Biswas, P. S., Prasad, B. and Khaleque Mian, M. A. (2002) Heterosis and genetic analysis in rice hybrid. Pakistan Journal of Biological Sciences, 5 (1), 1-5.

SAS Institute (2008) The SAS System for Windows, release 9.2. Cary NC: SAS Institute.

Shrotriya, G.C. (1998) Balanced Fertilizer-India Experience. Proceedings of Symposium on Plant Nutrition Management for Sustainable Agricultural Growth, NFDC, 8-10 December 1997, Islamabad. 
Steel, G. D. and Torrie, J. H. (1981) "Principles and Procedures of Statistics" (2 ${ }^{\text {nd }}$ ed.). McGraw-Hill Book Company. Inc. N. Y. xxi-633pp.

Steel, R. G. D., Torrie, J. H. and Dickey, D .A. (1997) "Principles and Procedures of Statistics": A Biometrical Approach”. The McGraw-Hill, Boston. 666p.

Tag El-Din, A. A., Hessein, E.M. and Ali, E. A. (2012) Path coefficient and correlation assessment of yield and yield associated traits in Sorghum (Sorghum bicolor L.) Genotypes. American-Eurasian J. Agric. \& Environ. Sci. 12 (6), 815-819,

White, P. J. and Brown, P. H. (2010) Plant nutrition for sustainable development and global health. Ann. Bot. 105, 1073-1080.

Young, K.J. and Long, S.P. (2000) Crop ecosystem responses to climatic change: Maize and sorghum. In: "Climate Change and Global Crop Productivity". Reddy, K.R, Hodges, H.F. (Ed.), CABI Publishing, Wallingford. 


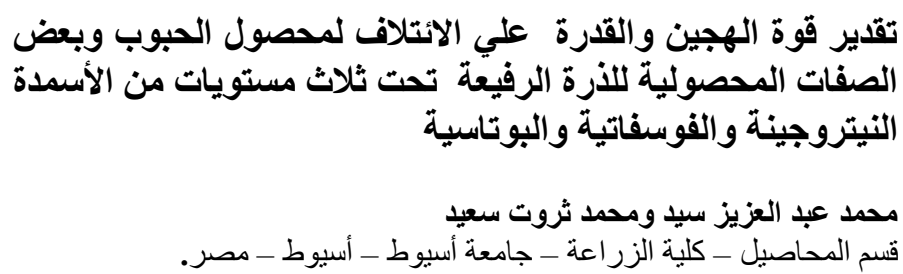

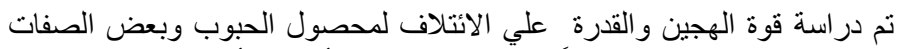



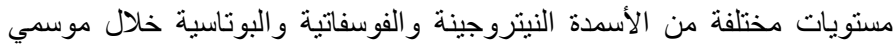



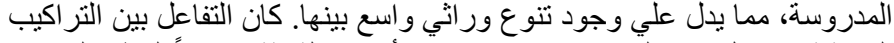

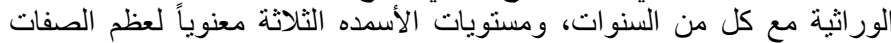

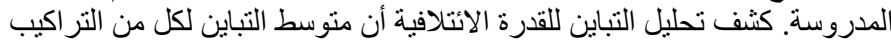

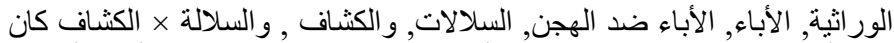

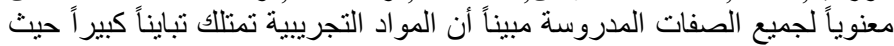

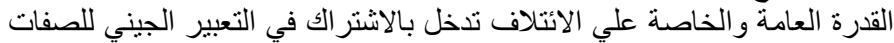

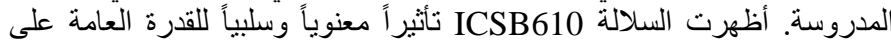

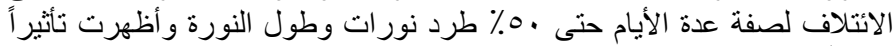

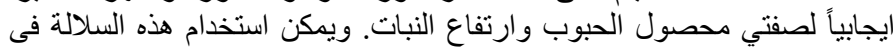

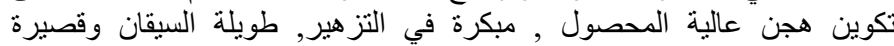

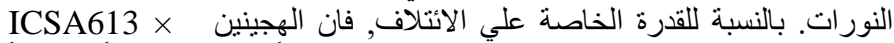

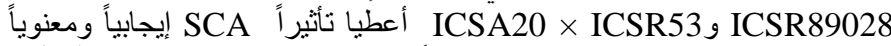





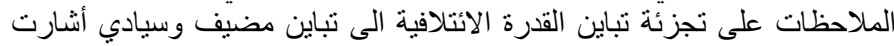

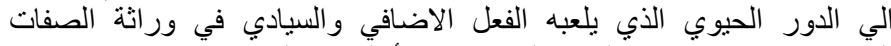

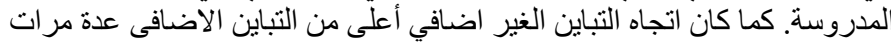

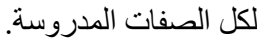

